\title{
Simultaneously Extraction of Caffeine and Chlorogenic Acid from Coffea canephora Bean using Natural Deep Eutectic Solvent-Based Ultrasonic Assisted Extraction
}

\author{
Adisya Miftah Syakfanaya', Fadlina Chany Saputri ${ }^{2}$, Abdul Mun'im ${ }^{3 *}$
}

\section{Adisya Miftah Syakfanaya ${ }^{1}$,} Fadlina Chany Saputri², Abdul Mun'im ${ }^{3 *}$

\section{${ }^{1}$ Faculty of Pharmacy, Universitas Indo- nesia, 16424, Depok, INDONESIA. ${ }^{2}$ Department of Pharmacology- Toxicology, Faculty of Pharmacy, Universitas Indonesia, 16424, Depok, INDONESIA. ${ }^{3}$ Department of Pharmacognosy- Phytochemistry, Faculty of Pharmacy, Universitas Indonesia, 16424, Depok, INDONESIA. \\ Correspondence \\ Dr. Abdul Mun'im, M.Si \\ Faculty of Pharmacy, Universitas Indonesia, Gedung A Rumpun IImu Kesehatan Lantai 3, Kampus UI Depok, 16424, West Java, INDONESIA. \\ Phone no : +62 8111184550 \\ E-mail:munim@farmasi.ui.ac.id \\ History \\ - Submission Date: 13-09-2018; \\ - Review completed: 02-10-2018; \\ - Accepted Date: 15-11-2018. \\ DOI : 10.5530/pj.2019.11.41}

Article Available online

http://www.phcogj.com/v11/i2

\section{Copyright}

(c) 2019 Phcog.Net. This is an openaccess article distributed under the terms of the Creative Commons Attribution 4.0 International license.

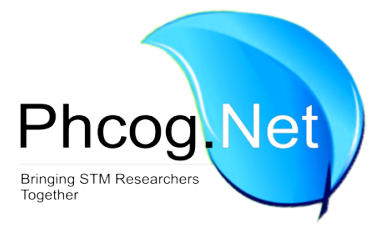

\begin{abstract}
Background: NADES is an alternative solvent in the extraction of metabolites from plants which has many environmental benefits, such as low toxicity, biodegradability, can dissolve polar and non-polar compounds, low costs and simple preparation. Objective: This study aims to determine the effect of natural deep eutectic solvent-based ultrasonic-assisted extraction (NADES-UAE) on enrichment of caffeine and chlorogenic acid in extract from green coffee beans (Coffea canephora). Methods: The powders were extracted using NADES-UAE method in several types of extraction condition, including the composition of NADES, water addition in NADES and extraction time. Caffeine and chlorogenic acid content were analyzed using HPLC, reverse phase system and $\mathrm{C}_{18}$ ODS-3 column. Results: The highest of caffeine and chlorogenic acid content was respectively, $7.89 \mathrm{mg} / \mathrm{g}$ and $28.62 \mathrm{mg} / \mathrm{g}$ (composition of NADES betaine: sorbitol [1:1.2] ratio and NADES-water addition [1:2] ratio for $30 \mathrm{~min}$ ). Conclusion: This research showed that the composition of NADES, extraction time and water addition are important parameter in extracting caffeine and chlorogenic acid content in green coffee beans.
\end{abstract}

Key words: Caffeine, Chlorogenic acid, Coffea canephora, Natural deep eutectic solvent, Ultrasonic assisted extraction.

\section{INTRODUCTION}

At present, the use of "green technology" has attracted the attention of researchers. Green technologies such as Ionic Liquid (IL) and Deep Eutectic Solvent (DES) are examples of developing green solvents that replace the use of organic solvents in many chemical processes such as extraction and synthesis. Deep Eutectic Solvents (DESs) are first introduced by Abbott et al. in 2003. ${ }^{1}$ DES is a mixture of two or more solid or liquid components which in certain composition have a high decrease in melting point so that it will form liquid at room temperature. ${ }^{2}$ If the DES compound is a primary metabolite, such as amino acids, organic acids, sugars or choline derivatives, DES is called Natural Deep Eutectic Solvents (NADES). NADES can be used as an alternative solvent in the extraction of natural materials because it has many advantages, including environmentally friendly, safe, forms liquids at room temperature (sometimes even below $0^{\circ} \mathrm{C}$ ), has a viscosity that can be adjusted easily, can dissolve polar and non-polar compounds, biodegradability, low toxicity, stable, low cost and simple preparation. ${ }^{2,3}$ Therefore, NADES is an attractive method to be used in the health sector, one of them is extracting green coffee beans.

Green coffee (Coffea canephora) robusta variant is one of the plants that has phenolic content in the form of chlorogenic acid and alkaloids in the form of caffeine which is higher than the arabica variant. Chlorogenic acid has pharmacological activity as an antioxidant, antibacterial, hepato-protective, cardio-protective, anti-inflammatory, anti-pireutic, neuro-protective, anti-obesity, antiviral, antimicrobial, anti-diabetic, antihypertensive, free radicals and stimulator of the central nervous system. ${ }^{4}$ Whereas caffeine has pharmacological activity in hypertension, stroke, heart failure, arrhythmias, sudden heart attacks and others related to vascular disease. ${ }^{5}$

In the previous study, extraction of green coffee beans was carried out using different NADES solvents compared organic solvents (acetone and citric acid solution) used the method of Dai et al. to the determination of total phenolic content in the form of gallic acid content. The result showed the content of gallic acid from NADES mixture was higher than organic solvents. ${ }^{2}$ Other studies also known, extraction of green coffee beans from Coffea arabica L. used NADES composition of lactic acid:sucrose produced caffeine and total phenolic in the form of gallic acid higher compared to citric acid: glucose. Analyzed with isocratic HPLC and the result showed, the highest caffeine content obtained was $4.45 \mathrm{mg} / \mathrm{g}$ 
(lactic acid: sucrose [4: 1]) and $1.30 \mathrm{mg} / \mathrm{g}$ (citric acid:glucose [2:1]). Whereas, the highest total phenolic content of the gallic acid obtained was $87.01 \mathrm{mg} / \mathrm{g}$ (lactic acid:sucrose [2: 1]) and $62.91 \mathrm{mg} / \mathrm{g}$ (citric acid: glucose [5:1]). ${ }^{6}$ In another study it was found that the extraction of Safflower plants that have aromatic pigments of varying polarity showed that most phenolic compounds were obtained with NADES with a yield of $75-97 \%$ ?

From various studies related to NADES, the use of betaine is still not widely used. Therefore, NADES research is needed using betain in other plants. Betaine is an example of NADES trimethyl derivatives of glycine. However, in previous studies it was known that the use of a mixture of betaine and sorbitol as a mixture of NADES with a ratio of 1:1.2 showed the results that the mixture was stable and optimum. NADES extraction results of a mixture of betaine and sorbitol showed higher extraction results than extraction using methanol. ${ }^{8}$ Until now, there have been no studies related to the extraction of caffeine and chlorogenic acid simultaneously using NADES. Therefore, this research will extract green coffee beans using NADES so that it is expected to obtain high caffeine and chlorogenic acid content.

\section{MATERIALS AND METHODS}

\section{Materials}

The sample of green coffee beans (Coffea canephora var. robusta) was obtained from a botanist of Harapan Jaya Farmers Group, Harapan Jaya Village, Way Ratai Subdistrict, Pesawaran District, Bandar Lampung City, Lampung Province. The coffee used which was ripe, marked with red coffee fruit and has been dried with a wet processing system. Then the sample was carried out by macroscopic determination at LIPI, Bogor. After that, the coffee beans were discarded and mashed with a certain size through the mesh 40 . Then, the coffee powder was stored in a tightly closed bottle which contains silica gel. Furthermore, stored in a closed cabinet at room temperature of $25^{\circ} \mathrm{C}$ and stored away from sharp-smelling material so as not to be damaged for further use. Caffeine standard (CSPC, China), chlorogenic acid standard (Wako Pure Chemical Industries, Japan), Betaine anhydrous (Shandong, China), sorbitol (Roqutte Freres, France), aqua demineralization and $70 \%$ ethanol (PT. Brataco, Indonesia). Acetonitrile, methanol HPLC grade, acetic acid glacial and urea (Merck, Germany). Aqua Pro Injection/API (PT. Ikapharmindo Putramas, Indonesia)

\section{Instrumentation}

Hotplate stirrer (IKA ${ }^{\oplus}$ C-MAG HS 4), analytical balance (VIBRA HT), High Performance Liquid Chromatography (Shimadzu LC 20 AD, Sil-20A-HT, CTO-20A, Kyoto, Japan), ultrasonic (Krisbow), HPLC column (Insertsil ODS3 5 $\mathrm{m}$ 4.6x150 $\mathrm{mm} \mathrm{C}_{18}$, GI Science, Tokyo, Japan), centrifuge (Universal 320) and micropipette 10-100 and 100-1000 $\mu$ l (Thermo Scientific, USA)

\section{Preparation Natural Deep Eutectic Solvent (NADES) Solvent}

NADES solution was formed of betaine and sorbitol mixture or betaine, sorbitol and urea mixture and was put in a beaker glass. Furthermore, the mixture was stirred using magnetic stirrer and heated over a hotplate stirrer at $80^{\circ} \mathrm{C}$ accompanied by agitation until a clear solution was formed. ${ }^{7}$

\section{Extraction Process of NADES-UAE}

The process used by Dai et al. and Duan et al. with slight modification. A number of samples were inserted into a closed bottle and a mixture of NADES was added (1:10). Next, the mixture was sonicated at a certain time. After that, it was transferred to a centrifuge tube and centrifuged at
$4500 \mathrm{rpm}$ for $17 \mathrm{~min}$, then filtered using a $0.45 \mu \mathrm{m}$ cellulose acetate filter. Then, the volume obtained was adjusted at the nearest volume. Furthermore, the suspension was diluted 10 times using aqua pro injection for analysis using HPLC. The extraction was carried out with three replications. ${ }^{7,8}$ The measurement parameter was obtained from the maximum yield value of chlorogenic acid and caffeine from several variations of the condition carried out. The extraction process was carried out in three different condition.

\section{Composition of NADES}

There were six kinds of NADES composition made consisted of betaine: sorbitol $(0.5: 1.2 ; 1: 1.2 ; 2: 1.2)$ and betaine: sorbitol: urea $(0.5: 1.2: 1 ; 1: 1.2: 1$; 2:1.2:1) and each was tested against the caffeine and chlorogenic acid content in green coffee beans. Extraction was carried out for $30 \mathrm{~min}$ with the amount of water in 50\% NADES. Then, the composition which showed optimum results was used as a solvent under further extraction condition.

\section{Water addition in NADES}

The water addition was varied in four types (25\%, 50\%, 75\% and 100\%). NADES used was the best composition in the first condition. Each was tested against caffeine and chlorogenic acid content in green coffee beans. Extraction was carried out for $30 \mathrm{~min}$. Extraction condition with optimum results was used on the next extraction condition.

\section{Extraction time}

The extraction time was differentiated in three types (15 $\mathrm{min}, 30 \mathrm{~min}$ and $60 \mathrm{~min}$ ). The best NADES in second extraction was used in this extraction condition. Each was tested against caffeine and chlorogenic acid content in green coffee beans.

\section{Standard Solution Preparation}

Standard solutions were prepared for caffeine and chlorogenic acid. Caffeine solution and chlorogenic acid were made into a stock solution with a concentration of $10 \mathrm{mg} / \mathrm{mL}$ prepared in $70 \%$ ethanol. From each of these stock solutions, a series solution was made with levels of $1 \times 10^{-4}$ $(0.1 \mu \mathrm{g} / \mathrm{ml})$ up to $1 \times 10^{-1}(100 \mu \mathrm{g} / \mathrm{ml})$. Furthermore, each series solution measured UV/Vis absorbance at $\lambda 272 \mathrm{~nm}$ for caffeine and $\lambda 326 \mathrm{~nm}$ for chlorogenic acid. Then the linear regression equation was calculated.

\section{Calibration Curve Preparation}

Calibration curves for the caffeine standard $10.000 \mathrm{ppm}(10 \mathrm{mg} / \mathrm{ml})$ and 3-caffeoylquinic acid (chlorogenic acid) standard $10.000 \mathrm{ppm}(10 \mathrm{mg} / \mathrm{ml})$ were obtained by injecting an increase in standard mixed concentration with a peak area measured at $272 \mathrm{~nm}$ wavelength for caffeine and $326 \mathrm{~nm}$ for 3-caffeoylquinic acid (chlorogenic acid). Each standard solution was diluted to $400 \mathrm{ppm}$. Then, a series solution was made with concentrations of $0.4,1,5,15,20,30$ and $80 \mathrm{ppm}$. Measurements were made three times. Furthermore, a complete spectrum will be obtained from 200 to $600 \mathrm{~nm}$ and extraction wavelengths to make chromatographic profiles (272 $\mathrm{nm}$ and $326 \mathrm{~nm}$, the maximum wavelength of each absorption for caffeine and CGA absorbance). The caffeine and chlorogenic acid content in each extract was identified by comparing the AUC (Area Under Curve or area under the curve) to the linear regression equation obtained from the series solution. ${ }^{9}$

\section{High Performance Liquid Chromatography (HPLC)} Analysis of Caffeine and Chlorogenic Acid Content

HPLC analysis used the Navarra et al. method with some modifications. Chromatographic measurements were carried out using Shimadzu HPLC, reverse system equipped with a pump (LC-20 AD), an auto-sampler 
(SIL-20A-HT), an automatic column temperature control oven (CTO-20A) and $20 \mu \mathrm{L}$ loop sample. The column used in the test was $\mathrm{C}_{18}$ column ODS, maintained at $25^{\circ} \mathrm{C}$ by an oven, was used for the measurement of the separation of caffeine and chlorogenic acid. The mobile phase used was a mixture of $0.1 \%$ acetic acid (v/v) (solvent $\mathrm{A}$ ) and acetonitrile (solvent B). The mobile phase was pumped at $1 \mathrm{~mL} / \mathrm{min}$ using the following gradient profile: from $90 \% \mathrm{~A}$ and $10 \% \mathrm{~B}$ for $20 \mathrm{~min}$ and then converted to $80 \% \mathrm{~A}$ and $20 \% \mathrm{~B}$ for $10 \mathrm{~min}$ isocratic and then returned to the initial condition (90\% A and 10\% B) for 5 min. ${ }^{9}$

\section{RESULTS AND DISCUSSION}

\section{Extraction Process}

In this study, the development of the extraction process was related to the development of green solvents in the form of NADES with the aim to obtain higher levels of secondary metabolites. NADES was a green solvent suitable for extraction of phenolic acids from plants compared to methanol which was toxic. Extraction condition were carried out on three different factors, such as NADES composition, water content in NADES and extraction time. Apart from choosing the best solvent, extraction was also affected by extraction condition, such as the selection of an extraction method with the UAE method. The UAE method was chosen because it related to time efficiency, the amount of solvent used, increased sample surface area, cost efficiency, easy to use, environmental friendliness and was available in the laboratory. In addition, the efficiency of the extraction method using UAE was because it can eliminate bubble cavities in NADES. Furthermore, the ultrasonic waves cause damage to plant cell walls resulting in the release of cell contents into the medium. However, these waves can also cause adverse effects such as degradation of phenolic content or produce free radicals in gas bubbles. ${ }^{10}$ In the UAE, the presence of mechanical agitation or ultrasonic can help increase the rate of movement of compounds in fluids.?

\section{Calibration curve}

The linear regression equation obtained for caffeine is the equation formula $\mathrm{y}=79580 \mathrm{x}-24933$ with $\mathrm{R}=0,999$ while chlorogenic acid with regression formula $y=46057 \mathrm{x}-26606$. Furthermore, it was used to calculate caffeine and chlorogenic acid in each extract. Where $y$ is concentration and $\mathrm{x}$ is peak area. These results were according with the theory. The HPLC profile can be seen in Figure 1.

\section{Caffeine and cholorogenic acid content determination Composition of NADES}

During the preparation process for NADES, HBA is a component that has an important role in the formation and stability of NADES. Compared to conventional solvents, the viscosity of NADES at room temperature limits the use of NADES because it causes slow mass transfer. ${ }^{8}$ To reduce

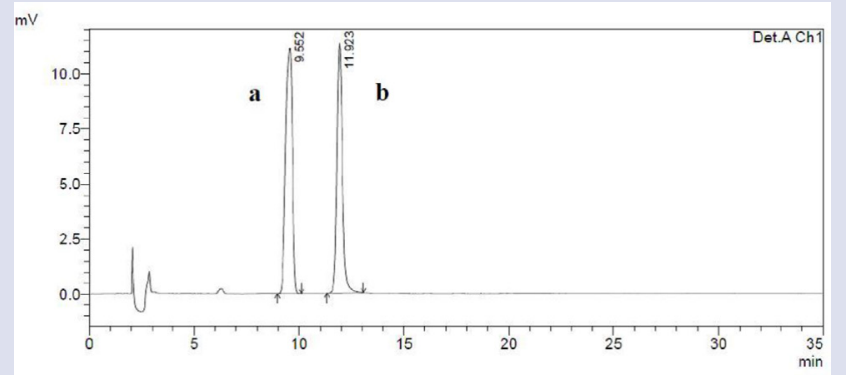

Figure 1: HPLC profile of caffeine (a) and chlorogenic acid standards (b).
Table 1: Results of effect of NADES composition on caffeine and chlorogenic acid content.

\begin{tabular}{ccccc}
\hline \multicolumn{3}{c}{ NADES Composition } & \multicolumn{2}{c}{ Yields (mg/g) } \\
\hline Betaine & Sorbitol & Urea & Caffeine & Chlorogenic acid \\
\hline 0.5 & 1.2 & - & 2.29 & 12.19 \\
1 & 1.2 & - & 5.14 & 13.46 \\
2 & 1.2 & - & 3.54 & 10.23 \\
0.5 & 1.2 & 1 & 3.28 & 2.81 \\
1 & 1.2 & 1 & 3.54 & 7.77 \\
2 & 1.2 & 1 & 2.86 & 6.48 \\
\hline
\end{tabular}

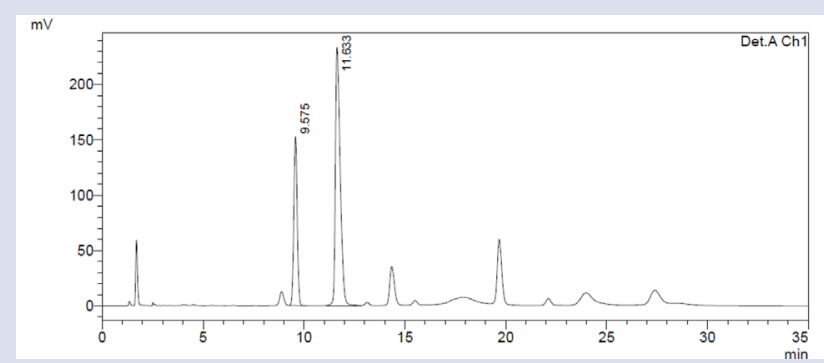

Figure 2: HPLC chromatogram of extract using NADES composition (betaine: sorbitol [1:1.2]).

the viscosity of NADES, the extraction condition can be adjusted by heating and adding a small amount of water. The results of each composition shown in Table 1.

The optimum result was shown by NADES with the composition of betain: sorbitol 1:1.2 with caffeine and chlorogenic acid yields, $5.14 \mathrm{mg} / \mathrm{g}$ and $13.46 \mathrm{mg} / \mathrm{g}$, respectively. The HPLC profile can be seen in Figure 2 . From these results indicate that an increase in the amount of betain affects the amount of caffeine and chlorogenic acid attracted. Betain is an HBA (hydrogen bond acceptor) that functions as a compound that receives hydrogen bonds while sorbitol is an HBD (hydrogen bond donor) that functions as a compound that donates hydrogen bonds. In addition, the presence of urea which is also HBD causes less caffeine and chlorogenic acid to be attracted. Previous studies showed extraction of phenolic content in the form of rosmarinic acid and salviaflaside from Prunella vulgaris using several different composition of NADES and with different mole ratios from 1:2 to 1:6. The results obtained in the composition of certain NADES with a mole ratio of 1:4 is the composition of NADES that gives the highest yield. Increased HBD concentration causes a significant reduction in the results of phenolic content extraction. In this study, extraction condition in various composition of NADES, NADES consisting of a mixture of Betain: Sorbitol: Urea had lower yields compared to NADES which consisted of only two composition (HBA and HBD, betain: sorbitol). This can be due to the presence of two types of HBD in the same composition. As known from previous studies, that an increase in HBD concentration causes a significant reduction in the results of phenolic content extraction. Extraction results with NADES betain and L-proline are higher than the results obtained from extraction using methanol. ${ }^{10}$

\section{Water addition in NADES}

Addition of water in NADES has a significant advantage in reducing viscosity. Another study showed the amount of extracted substances will increase according to the increase in the percentage of water until the 
Table 2: Results of effect of water addition on caffeine and chlorogenic acid content.

\begin{tabular}{ccc}
\hline \multirow{2}{*}{$\begin{array}{c}\text { Water Addition } \\
(\%)\end{array}$} & \multicolumn{2}{c}{ Yields $(\mathrm{mg} / \mathrm{g})$} \\
\cline { 2 - 3 } & Caffeine & Cholorogenic acid \\
\hline 25 & 0.60 & 1.68 \\
50 & 2.60 & 8.64 \\
75 & 4.26 & 16.42 \\
100 & 7.89 & 28.62 \\
\hline
\end{tabular}

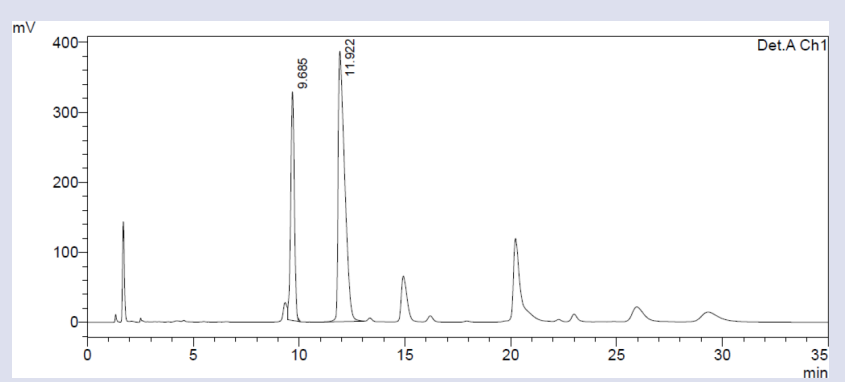

Figure 3: HPLC chromatogram of extract from addition of $100 \%$ water in NADES composition.

water concentration reaches $35 \%$. However, if the percentage of water was too high, it will reduce the alkaloid extraction results, related to the decrease in the interaction between DES and alkaloids with an increase in the polarity of the mixture. ${ }^{8}$ The results of each water addition shown in Table 2. The highest results was shown by NADES of $100 \%$ water addition (1:2), with caffeine and chlorogenic acid yields $7.89 \mathrm{mg} / \mathrm{g}$ and $28.62 \mathrm{mg} / \mathrm{g}$, respectively. The HPLC profile can be seen in Figure 3. At the time of preparing NADES, many composition needed to be added with a little water in it, because with the addition of water will reduce the viscosity of NADES and increase its conductivity to 100 times in some NADES. In the presence of water causes gradually weakening the hydrogen bonding interaction between the two NADES, even disappearing completely at $50 \%(\mathrm{v} / \mathrm{v})$ water addition. ${ }^{11}$

The greater amount of water added in the mixture of NADES, the greater number of target compounds attracted. This is because the water content in NADES also has a large influence on the extraction results, varies greatly according to the target compound and the NADES used. NADES with high water content was better for extracting polar compounds and NADES with low water content suitable for extraction of less polar compounds. ${ }^{7}$ In another study it was stated that the use of DES in the extraction of phenolic acids in the form of chlorogenic acid showed that the extraction of 1,6-3,6 was higher when there was a $10 \%$ addition of water to the composition of NADES. ${ }^{10}$ From this optimization, it can be seen that the water concentration in the NADES-water mixture and in the solid-liquid ratio is the main parameter that gives effect to the extraction results.

\section{Extraction time}

The time of extraction was the efficiency factor of extraction using UAE. The longer of extraction time causes will increase the temperature in the extraction media.

The results of each extraction time shown in Table 3. The optimum result was shown by NADES with an extraction time of 30 min with caffeine and chlorogenic acid yields of $4.85 \mathrm{mg} / \mathrm{g}$ and $13.06 \mathrm{mg} / \mathrm{g}$, respectively. The HPLC profile can be seen in Figure 4. As known, chlorogenic acid
Table 3: Results of effect of extraction time on caffeine and chlorogenic acid content.

\begin{tabular}{ccc}
\hline \multirow{2}{*}{$\begin{array}{c}\text { Extraction Time } \\
\text { (minutes) }\end{array}$} & \multicolumn{2}{c}{ Yields $(\mathrm{mg} / \mathrm{g})$} \\
\cline { 2 - 3 } & Caffeine & Chlorogenic acid \\
\hline 15 & 2.89 & 6.48 \\
30 & 4.85 & 13.06 \\
60 & 4.23 & 9.72 \\
\hline
\end{tabular}

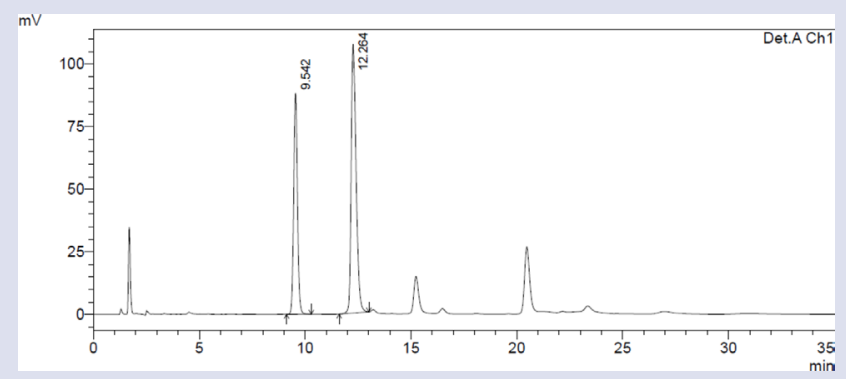

Figure 4: HPLC chromatogram of extract using NADES with $30 \mathrm{~min}$ extraction time.

is thermolable, so that the longer the extraction time will increase the temperature in the extraction container and the heat transfer caused the chlorogenic acid to be degraded. In previous studies, it was known, extraction $>90 \mathrm{~min}$ at $55^{\circ} \mathrm{C}$ did not significantly increase extraction results. Extraction using NADES Betain-1,4 Butanediol of phenolic content in the form of vitexin was known at $40^{\circ} \mathrm{C}$ with a $30 \mathrm{~min}$ to produce levels of $17.32 \mathrm{ppm}$ and at $60 \mathrm{~min}$ there was no significant increase, namely $17,43 \mathrm{ppm}$. While at $55^{\circ} \mathrm{C}$ with extraction time of $30 \mathrm{~min} 111.20$ and at $60 \mathrm{~min}$ decreased to $107,52 .{ }^{12}$

From all the results above, it can be seen that chlorogenic acid content is higher than caffeine content in green coffee beans robusta variant. Chlorogenic acid has many subgroups of isomers, including caffeoylquinic acids (CQA has three isomers: 3-, 4- and 5-CQA), dicaffeoylquinic acids (diCQAs has three isomers: 3,4-, 3,5- and 4, 5-diCQA) and feruloylquinic acids (FQAs have three isomers: 3-, 4- and 5-FQA). ${ }^{13}$ Previous research has suggested that the amount of chlorogenic acid in each brewed coffee was different, for example in several studies reported, 5-CQA was the most important component of chlorogenic acid in brewed coffee and in green coffee bean extracts. ${ }^{14-18}$

\section{CONCLUSION}

Based on the results above, it can be seen that the effect of NADES composition, extraction time and water content in NADES is an important matter in extracting caffeine and chlorogenic acid content in green coffee beans using NADES-UAE. From this study it can be seen that NADES is compatible and can be applied to cosmetic manufacture as well as dermatologist, drug formulation or used in food production.

\section{ACKNOWLEDGEMENT}

This research supported by Universitas Indonesia for Hibah PITTA 2018.

\section{CONFLICT OF INTEREST}

The authors declare no conflict of interest. 


\section{ABBREVIATIONS}

HPLC: High-performance liquid chromatography; DES: Deep eutectic solvent; NADES: Natural deep eutectic solvent; ODS: Octadecyl-silica; UAE: Ultrasonic-assisted extraction.

\section{REFERENCES}

1. Aroso IM, Paiva A, Reis RL, Rita A, Duarte C. Natural deep eutectic solvents from choline chloride and betaine - Physicochemical properties. J Mol Liq. 2017:241:654-61.

2. Paiva A, Craveiro R, Aroso I, Martins M, Reis RL, Duarte ARC. Natural Deep Eutectic Solvents-Solvents for the $21^{\text {st }}$ Century. 2014.

3. Espino M, Fernández MDLÁ, Gomez FJV, Silva MF. Natural designer solvents for greening analytical chemistry. Trends in Analytical Chemistry. 2016;76:126-36.

4. Naveed M, Hejazi V, Abbas M, Ali A, Jilany G, Shumzaid M, et al. Biomedicine and Pharmacotherapy Chlorogenic acid (CGA): A pharmacological review and call for further research. Biomedicine and Pharmacotherapy. 2018;97:67-74.

5. Turnbull D, Rodricks J, Mariano V, Chowdhury GF. Caffeine and cardiovascular health. Regulatory Toxicology and Pharmacology. 2017;89:165-85.

6. Ahmad I, Pertiwi AS, Kembaren YH, Rahman A, Mun'im A. Application of Natural Deep Eutectic Solvent-Based Ultrasonic Assisted Extraction of Total Polyphenolic and Caffeine Content from Coffe Beans (Coffea beans L.) For Instant Food Products. Journal of Applied Pharmaceutical Science. 2018;8(8):138-43.

7. Dai Y, Van S J, Witkamp G. Analytica Chimica Acta Natural deep eutectic solvents as new potential media for green technology. Anal Chim Acta. 2013;766:61-8.

8. Duan L, Dou L, Guo L, Li P, Liu E. Comprehensive evaluation of deep eutectic solvents in extraction of bioactive natural products: Comprehensive evaluation of deep eutectic solvents in extraction of. American Chemical Society Publications. 2016:4(4):2405-11.
9. Navarra G, Moschetti M, Guarrasi V, Mangione MR, Militello V, Leone M. Simultaneous Determination of Caffeine and Chlorogenic Acids in Green Coffee by UVNis Spectroscopy. Journal of Chemistry. 2017.

10. Ruesgas-Ramon M, Figueroa-espinoza MC, Durand E. Application of Deep Eutectic Solvents (DES) for Phenolic Compounds Extraction: Overview, Challenges and Opportunities. Journal of Agricultural and Food Chemistry. 2017;65(18):3591-601.

11. Dai $Y$, Witkamp G, Verpoorte R, Choi $Y H$. Tailoring properties of natural deep eutectic solvents with water to facilitate their applications. Food Chem. 2015;187:14-9.

12. Mulia K, Muhammad F, Krisanti E. Extraction of Vitexin from Binahong (Anredera cordifolia (Ten.) Steenis) Leaves using Betaine - 1, 4 Butanediol Natural Deep Eutectic Solvent (NADES). American Institute of Physics. 2017;1823(1):20018.

13. Jeon J, Kim H, Jeong I, Hong S, Oh M, Park K, et al. Determination of chlorogenic acids and caffeine in homemade brewed coffee prepared under various conditions. Journal of Chromatography B. 2017;1064:115-23.

14. Farah A, de Paulis T, Trugo LC, Martin PR. Effect of roasting on the formation of chlorogenic acid lactones in coffee. J Agric Food Chem. 2005;53(5):1505-13.

15. Fujioka K, Shibamoto T. Chlorogenic acid and caffeine contents in various commercial brewed coffees. Food Chem. 2008;106(1):217-21.

16. Perrone D, Farah A, Donangelo CM, de Paulis T, Martin PR. Comprehensive analysis of major and minor chlorogenic acids and lactones in economically relevant Brazilian coffee cultivars. Food Chem. 2008;106(2):859-67.

17. Moon JK, Yoo HS, Shibamoto T. Role of roasting condition in the level of chlorogenic acid content in coffee beans: correlation with coffee acidity. J Agric Food Chem. 2009;57(12):5365-9.

18. Craig AP, Fields C, Liang N, Kitts D, Erickson A. Performance review of a fast HPLC-UV method for the quantification of chlorogenic acids in green coffee bean extracts. Talanta. 2016;154:481-5.

\section{GRAPHICAL ABSTRACT}

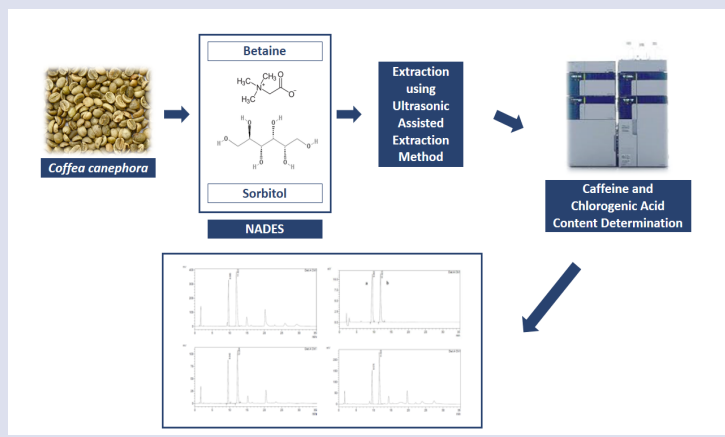

\section{SUMMARY}

- NADES-UAE of Betaine-Sorbitol can extract the biomarker compound from green coffee bean. Betain-Sorbitol (1:1.2) ratio, NADES-water addition (1:2) ratio for 30 min showed the highest caffeine and chlorogenic acid content.

\section{ABOUT AUTHORS}

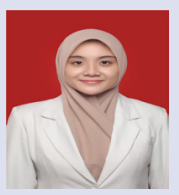

Adisya Miftah Syakfanaya: its a master student at Department of Pharmaceutical Sciences, Faculty of Pharmacy, Universitas Indonesia (UI) Depok, West Java, Indonesia. The master research focused on the study of Natural Deep Eutectic Solvent (NADES) and development of extraction method.

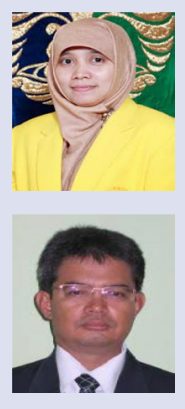

Fadlina Chany Saputri : is an Associate Professor at Department Pharmacology-Toxicology, Faculty of Pharmacy, Universitas Indonesia (UI) Depok, West Java, Indonesia. Her research focused on herbal plants and its activity on degenerative disease (such as diabetes mellitus, antihypertension and other cardiovascular disease)

Abdul Mun'im: Ph.D., is an Associate Professor at Department Pharmaceutical Sciences, Faculty of Pharmacy Universitas Indonesia (UI) Depok, West Java, Indonesia. He has experience in the area of Pharmacognosy and Phytochemistry, working in drugs discovery of herbal plants, extraction technology, metabolite profiling, structure elucidation, and degenerative disease (such as diabetes mellitus, antihypertension and cholesterol).

Cite this article: Syakfanaya AM, Saputri FC, Mun'im A. Simultaneously Extraction of Caffeine and Chlorogenic Acid from Coffea canephora Bean using Natural Deep Eutectic Solvent-Based Ultrasonic Assisted Extraction. Pharmacog J. 2019;11 (2):267-71. 